\title{
Determinants of the circadian blood pressure pattern in hospitalized hypertensive patients
}

\author{
Martyna Zaleska', Olga Możeńska', Agnieszka Segiet', Jan Gierattowski', Monika Petelczyc², \\ Zbigniew Dubielski', Dariusz A. Kosior ${ }^{3}$ \\ 'Department of Cardiology and Hypertension, Central Research Hospital, the Ministry of the Interior and Administration, Warsaw, Poland \\ ${ }^{2}$ Faculty of Physics, Warsaw University of Technology, Warsaw, Poland \\ ${ }^{3}$ Department of Applied Physiology, Mossakowski Institute of Experimental and Clinical Medicine, Polish Academy of Sciences, Warsaw, Poland
}

\begin{abstract}
Background. Non-dipping hypertension might be associated with increased cardiovascular risk and multiple diseases. The aim of our study was to assess if there are parameters identified in 24-hour ECG-Holter monitoring (ECG-Holter), transthoracic echocardiography (TTE), ECG parameters or laboratory data that allow prediction of circadian blood pressure profile (CBPP).

Material and methods. One hundred and three consecutive patients (male: 50.5\%), who underwent 24-hour ambulatory BP measurement and ECG-Holter simultaneously were analyzed. We divided patients into 3 groups: dipping was defined as $10-20 \%(28.2 \%)$, non-dipping as < 10\% (50.5\%) fall in nocturnal BP and reverse-dipping as higher nocturnal than diurnal BP $(21.4 \%)$. Additionally, we performed TTE and laboratory check-up in all patients. We built multivariable models for nocturnal fall in systolic BP (SBP) and CBPP.

Results. Multivariable model based on clinical factors was: nocturnal fall in SBP $(\%)=[13.28-0.11 \times$ age $-8.33 \times(\mathrm{di}-$ lated cardiomyopathy) $-5.95 \times \mathrm{PAD}-6.02 \times \alpha$-adrenolytic]. Multivariable model based on laboratory, echocardiographic and electrocardiographic parameters was: nocturnal fall in SBP $(\%)=[-27.28+1.47 \times$ hemoglobin $-0.14 \times \mathrm{CK}-\mathrm{MB}+0.14 \times$ maximal heart rate]. Multivariable model for CBPP based on clinical factors included use of $\beta$ - or $\alpha$-adrenolytics or torasemide.

Conclusions. We proved that nocturnal fall in SBP and CBPP could be predicted based on ECG-Holter parameters, laboratory data and TTE results, as well as based on detailed medical history. These findings may have implications on care of patients with hypertension.

Key words: ambulatory blood pressure monitoring; circadian rhythm; hypertension; hypotensive therapy
\end{abstract}




\section{Introduction}

Hypertension is a growing problem, and currently, there are over 1 billion hypertensive individuals worldwide $[1,2]$. Some diseases, such as autonomic neuropathies, obstructive sleep apnea, diabetes, autonomic dysfunction, and chronic kidney disease (CKD), also seem to relate to lower fall in nocturnal blood pressure (BP) [3, 4]. Patients affected with secondary or malignant hypertension also have higher prevalence of non-dipping pattern of circadian blood pressure profile (CBPP) [3, 4].

Recently published study suggests that asleep blood pressure is the most important risk factor for cardiovascular events which can be derived from BP values. In the study of Hermida et al., the asleep systolic BP was the most important risk factor for the primary outcome (defined as composite of cardiovascular death, myocardial infarction, coronary revascularization, heart failure and stroke) during 5.1-year median follow-up, and it was regardless of office and awake systolic BP values. The authors emphasize also the fact that attenuation of above-mentioned parameter was the most significant marker of eventfree survival [5].

Despite all above-mentioned facts the availability of 24-hour ambulatory blood pressure monitoring (ABPM) is rather low. The awareness about these novel goals in hypertension treatment is also small. Furthermore, little is known about factors either determining or allowing predicting CBPP, besides above-mentioned comorbidities. The aim of the study was to clarify whether there are such factors among 24-hour ECG Holter monitoring (ECG-Holter), transthoracic echocardiography (TTE), ECG parameters or laboratory data.

\section{Material and methods}

\section{Study population}

This was a retrospective study analyzing data of 103 patients hospitalized in the Department of Cardiology and Hypertension in Central Research Hospital of the Ministry of Interior in Warsaw between January 2012 and December 2013. Consecutive hypertensive patients, who simultaneously underwent ABPM and ECG-Holter, were included into analysis. According to nocturnal BP fall pattern we divided the patients into three groups: dippers, non-dippers, reversedippers. Dipping was defined as a $10-20 \%$ fall in nocturnal systolic BP (SBP), non-dipping as less than $10 \%$, and reverse-dipping as $0 \%$ at most fall in nocturnal SBP $[6,7]$. Collected data were analyzed retro- spectively and Local Ethics Committee gave consent to conduct the study. All procedures were performed in accordance with the Declaration of Helsinki on the treatment of human subjects.

\section{Measurements}

We used Tracker NIBP2 SpaceLabs Healthcare and ABP 90217-7Q SpaceLabs Healthcare devices to obtain ABPM and Lifecard CF Reynolds Medical device to assess ECG-Holter. Measurements of BP were performed every 10 minutes during awake hours and every 30 minutes during the night-time. Additionally, all patients underwent TTE with Philips iE-33 and EPIQ ultrasound machines.

\section{Statistical analysis}

Statistical analysis was performed with $\mathrm{R}$ version 3.1.2 [8]. Continuous variables are presented as number of observations and mean with standard deviation, categorical variables are reported as frequencies and percentages. The distribution of continuous variables was first analyzed with Shapiro-Wilk test of normality and then, according to the results, ANOVA test or Kruskal-Wallis test were used. Categorical variables were compared using Fisher's exact test. The significance level was set at 0.05 . The impact of clinical, laboratory, echocardiographic and electrocardiographic factors on nocturnal fall in SBP and CBPP was analyzed with linear regression analysis and ordinal logistic regression analysis, respectively. Univariable and mutlivariable analyses were performed. Two multivariable models were built, with independent variables chosen from clinical factors and from laboratory, echocardiographic and electrocardiographic parameters for each dependent variable (nocturnal fall in SBP and CBPP). Variables considered in multivariable models were chosen from the set of variables with Wald's test p-value less than 0.25 in univariable analysis and that were classified in expert analysis as having potential influence on dependent variables. Multivariable models were selected with backward elimination procedure. Linear regression coefficients or proportional odds ratios were reported, with 95\% confidence interval and Wald's test p-values, respectively.

\section{Results}

One hundred three consecutive patients (male: $50.5 \%)$ with mean age $63.9( \pm 17.7)$ years who simultaneously underwent ABPM and ECG-Holter were included in further analysis. According to ABPM outcomes 29 (28\%) patients were classified as 
dippers, $52(50 \%)$ as non-dippers and consecutive 22 $(21 \%)$ as reverse-dippers. The reverse-dipper group was the oldest, with mean age $74.9 \pm 10.9$ years $(\mathrm{p}<$ 0.001 ). Study population characteristics are presented in Table I. There were significant differences between groups in occurrence of CKD and peripheral artery disease $(\mathrm{PAD})$, with higher prevalence of those diseases in non-dipper and the highest in reversedipper group. In Table I we also included comparison of drugs taken by patients' subgroups. We found that reverse-dippers were most likely to take both: $\beta$ - and $\alpha$-adrenolytics as well as torasemide. Table I also includes mean values of diurnal and nightly, both systolic and diastolic, BP. In Table II we present laboratory as well as TTE and in Table III ECG-Holter parameters.
The multivariable model for nocturnal fall in SBP based on clinical factors was:

$$
\begin{gathered}
\text { Nocturnal fall in SBP }[\%]=13.28-0.11 \times \text { age } \\
-8.33 \times D C M-5.95 \times P A D \\
-6.02 \times \alpha \text {-adrenolytic }
\end{gathered}
$$

According to the presented model, if a patient's age increases by 1 year, nocturnal fall in SBP decreases by $0.11 \%$ [ $95 \%$ confidence interval (CI): -0.187 to -0.029 ], if a patient has dilated cardiomyopathy (DCM), PAD or uses $\alpha$-adrenolytics, nocturnal fall in SBP decreases by $8.33 \%$ (95\% CI: -15.836 to $-0.823), 5.95 \%$ (95\% CI: -10.540 to -1.361$)$ and 6.02\% (95\% CI: -10.010 to -2.024$)$, respectively

\begin{tabular}{|c|c|c|c|c|}
\hline & $\begin{array}{l}\text { Dipper } \\
\text { (29 pts) }\end{array}$ & $\begin{array}{l}\text { Non-dipper } \\
\text { (52 pts) }\end{array}$ & $\begin{array}{c}\text { Reverse-dipper } \\
\text { (22 pts) }\end{array}$ & \\
\hline Age (years) & $55.24 \pm 17.83$ & $63.98 \pm 17.46$ & $74.91 \pm 10.86$ & $\mathrm{p}<0.001$ \\
\hline Gender (M/F) & $16 / 13$ & $30 / 22$ & $6 / 16$ & $p=0.05$ \\
\hline SBP day [mm Hg] & $129.72 \pm 11.30$ & $127.48 \pm 13.81$ & $125.77 \pm 15.32$ & $p=0.58$ \\
\hline SBP night [mm Hg] & $111.55 \pm 9.16$ & $121.58 \pm 13.73$ & $133.00 \pm 18.04$ & $p<0.001$ \\
\hline SBP fall & $13.93 \pm 2.83$ & $4.63 \pm 2.91$ & $-5.61 \pm 3.71$ & $p<0.001$ \\
\hline DBP day [mm Hg] & $74.41 \pm 8.19$ & $71.23 \pm 8.67$ & $67.45 \pm 6.53$ & $p=0.009$ \\
\hline DBP night [mm Hg] & $61.34 \pm 6.67$ & $65.29 \pm 7.87$ & $67.86 \pm 8.05$ & $p=0.009$ \\
\hline DBP fall & $17.46 \pm 3.93$ & $8.21 \pm 5.32$ & $-0.53 \pm 5.35$ & $p<0.001$ \\
\hline $\mathrm{HF}$ & $8(27.59 \%)$ & $17(32.69 \%)$ & $11(52.38 \%)$ & $p=0.19$ \\
\hline HF-REF & $0(0.00 \%)$ & $1(1.92 \%)$ & $0(0.00 \%)$ & $p>0.999$ \\
\hline HF-PEF & $6(20.69 \%)$ & $15(28.85 \%)$ & $10(47.62 \%)$ & $p=0.13$ \\
\hline CKD & $1(3.70 \%)$ & $9(17.65 \%)$ & $6(30.00 \%)$ & $p=0.05$ \\
\hline PAD & $1(4.00 \%)$ & $3(5.88 \%)$ & $5(26.32 \%)$ & $p=0.03$ \\
\hline DCM & $0(0.00 \%)$ & $2(3.85 \%)$ & $2(10.00 \%)$ & $p=0.18$ \\
\hline OSA & $7(25.00 \%)$ & $13(25.00 \%)$ & $2(9.09 \%)$ & $p=0.3$ \\
\hline DM & $4(14.29 \%)$ & $7(13.73 \%)$ & $2(10.00 \%)$ & $p>0.999$ \\
\hline $\mathrm{AF}$ & $3(10.34 \%)$ & $15(29.41 \%)$ & $8(36.36 \%)$ & $p=0.06$ \\
\hline$\beta$-adrenolytics & $15(57.69 \%)$ & $32(69.57 \%)$ & $19(95.00 \%)$ & $p=0.01$ \\
\hline$\alpha$-adrenolytics & $0(0.00 \%)$ & $6(13.04 \%)$ & $6(30.00 \%)$ & $p=0.009$ \\
\hline Ca-antagonists & $9(34.62 \%)$ & $26(56.52 \%)$ & $9(45.00 \%)$ & $p=0.2$ \\
\hline ACE-I & $16(61.54 \%)$ & $22(47.83 \%)$ & $13(65.00 \%)$ & $p=0.39$ \\
\hline ARB & $3(11.54 \%)$ & $14(30.43 \%)$ & $6(30.00 \%)$ & $p=0.16$ \\
\hline Aldosterone antagonists & $0(0.00 \%)$ & $7(15.22 \%)$ & $1(5.00 \%)$ & $p=0.07$ \\
\hline Furosemide & $0(0.00 \%)$ & $8(17.39 \%)$ & $2(10.00 \%)$ & $p=0.06$ \\
\hline Torasemide & $1(3.85 \%)$ & $2(4.35 \%)$ & $7(35.00 \%)$ & $p=0.002$ \\
\hline
\end{tabular}
(Tab. IV).

Table I. Population characteristics

pts - patients; $p$ - overall p-value for 3-group comparison of means (ANOVA test) or distributions (Kruskal-Wallis test) for continuous variables and percentages $\left(\chi^{2}\right.$ test) for categorical variables; SBP — systolic blood pressure; DBP - diastolic blood pressure; HF — heart failure; HF-REF - HF with restricted ejection fraction; HF-PEF - HF with preserved ejection fraction; CKD — chronic kidney disease; PAD - peripheral artery disease; DCM — dilated cardiomyopathy; OSA - obstructive sleep apnea syndrome; DM — diabetes mellitus; AF — atrial fibrillation; ACE-I — angiotensin-converting-enzyme inhibitor; ARB — angiotensin II receptor blocker 
Table II. Laboratory and echocardiographic findings

\begin{tabular}{|c|c|c|c|c|}
\hline & $\begin{array}{l}\text { Dipper } \\
\text { (29 pts) }\end{array}$ & $\begin{array}{l}\text { Non-dipper } \\
\text { (52 pts) }\end{array}$ & $\begin{array}{l}\text { Reverse-dipper } \\
\text { (22 pts) }\end{array}$ & \\
\hline $\mathrm{HGB}[\mathrm{g} / \mathrm{dL}]$ & $14.30 \pm 1.42$ & $13.84 \pm 1.57$ & $12.70 \pm 1.40$ & $p=0.002$ \\
\hline $\mathrm{RBC}\left[\times 10^{6} / \mu \mathrm{L}\right]$ & $4.81 \pm 0.56$ & $4.61 \pm 0.51$ & $4.28 \pm 0.46$ & $p=0.003$ \\
\hline HCT (\%) & $42.24 \pm 3.82$ & $41.57 \pm 4.15$ & $39.04 \pm 3.13$ & $p=0.02$ \\
\hline PLT $\left[\times 10^{9} / \mathrm{L}\right]$ & $207.75 \pm 46.43$ & $199.96 \pm 58.39$ & $212.40 \pm 62.87$ & $p=0.68$ \\
\hline $\operatorname{MCV}[\mathrm{fL}]$ & $88.21 \pm 3.73$ & $89.97 \pm 4.70$ & $91.84 \pm 6.20$ & $p=0.09$ \\
\hline $\mathrm{MCH}[\mathrm{pg}]$ & $29.76 \pm 1.17$ & $29.99 \pm 1.73$ & $30.02 \pm 1.97$ & $p=0.81$ \\
\hline $\mathrm{MCHC}[\mathrm{g} / \mathrm{dL}]$ & $33.66 \pm 1.16$ & $33.36 \pm 0.96$ & $32.73 \pm 0.97$ & $p=0.001$ \\
\hline Urea [mg/dL] & $30.21 \pm 5.85$ & $41.04 \pm 32.72$ & $46.71 \pm 21.11$ & $p=0.03$ \\
\hline eGFR $\left[\mathrm{mL} / \mathrm{min} / 1.73 \mathrm{~m}^{2}\right]$ & $88.15 \pm 18.77$ & $80.35 \pm 26.42$ & $76.35 \pm 29.04$ & $p=0.25$ \\
\hline Creatinine $[\mathrm{mg} / \mathrm{dL}]$ & $0.84 \pm 0.17$ & $1.15 \pm 1.70$ & $0.93 \pm 0.44$ & $p=0.47$ \\
\hline AST [U/L] & $20.86 \pm 5.89$ & $26.02 \pm 14.03$ & $29.88 \pm 30.01$ & $p=0.4$ \\
\hline ALT [U/L] & $25.30 \pm 13.02$ & $31.49 \pm 30.46$ & $23.59 \pm 30.22$ & $p=0.02$ \\
\hline NT-proBNP [pg/mL] & $332.40 \pm 359.33$ & $1490.38 \pm 2507.92$ & $1414.30 \pm 2919.21$ & $p=0.65$ \\
\hline CK $[\mu \mathrm{g} / \mathrm{L}]$ & $109.56 \pm 38.57$ & $92.66 \pm 49.09$ & $119.62 \pm 65.23$ & $p=0.1$ \\
\hline CK-MB $[\mu \mathrm{g} / \mathrm{L}]$ & $16.56 \pm 5.09$ & $18.75 \pm 9.88$ & $24.82 \pm 16.82$ & $p=0.03$ \\
\hline $\mathrm{EF}(\%)$ & $62.39 \pm 4.69$ & $61.35 \pm 7.23$ & $59.86 \pm 6.23$ & $p=0.13$ \\
\hline LVDd [mm] & $50.31 \pm 4.43$ & $50.18 \pm 5.88$ & $50.10 \pm 5.94$ & $p=0.7$ \\
\hline RVDd [mm] & $31.50 \pm 4.53$ & $32.73 \pm 4.86$ & $31.45 \pm 6.11$ & $p=0.48$ \\
\hline LAD [mm] & $40.21 \pm 4.80$ & $43.75 \pm 6.15$ & $41.76 \pm 5.22$ & $p=0.03$ \\
\hline IVSd [mm] & $10.59 \pm 1.21$ & $10.94 \pm 1.77$ & $10.11 \pm 3.00$ & $p=0.68$ \\
\hline PWDd [mm] & $10.34 \pm 1.34$ & $10.76 \pm 1.73$ & $10.14 \pm 1.53$ & $p=0.26$ \\
\hline TAPSE [mm] & $22.00 \pm 4.24$ & $23.00 \pm 5.89$ & $21.83 \pm 2.99$ & $p=0.9$ \\
\hline IVC [mm] & $16.00 \pm 4.58$ & $20.50 \pm 5.45$ & $15.46 \pm 7.98$ & $p=0.66$ \\
\hline \multicolumn{5}{|l|}{ VHD } \\
\hline$A R$ & $7(25.00 \%)$ & $18(35.29 \%)$ & $6(28.57 \%)$ & $p=0.63$ \\
\hline AS & $0(0.00 \%)$ & $2(3.92 \%)$ & $0(0.00 \%)$ & $p=0.71$ \\
\hline MR & $20(68.97 \%)$ & $36(70.59 \%)$ & 18 (85.71\%) & $p=0.37$ \\
\hline MS & $0(0.00 \%)$ & $0(0.00 \%)$ & $0(0.00 \%)$ & NA \\
\hline TR & $10(35.71 \%)$ & $28(54.90 \%)$ & $12(57.14 \%)$ & $p=0.24$ \\
\hline TS & $0(0 \%)$ & 2 (3.92\%) & $0(0.00 \%)$ & $p=0.71$ \\
\hline $\mathrm{PR}$ & $0(0.00 \%)$ & $1(1.96 \%)$ & $1(4.76 \%)$ & $p=0.45$ \\
\hline PS & $0(0.00 \%)$ & $0(0.00 \%)$ & $0(0.00 \%)$ & NA \\
\hline
\end{tabular}

pts - patients; $p$ - overall p-value for 3-group comparison of means (ANOVA test) or distributions (Kruskal-Wallis test) for continuous variables; HGB — hemoglobin; RBC — red blood cells count; HCT — hematocrit; $\mathrm{PLT}$ - platelets count; $\mathrm{MCV}$ - mean corpuscular volume; $\mathrm{MCH}$ - mean corpuscular hemoglobin; $\mathrm{MCHC}$ - mean corpuscular hemoglobin concentration; eGFR — estimated glomerular filtration rate; CRP - C-reactive protein; AST — aspartate transaminase; ALT — alanine transaminase; NT-proBNP - N-terminal of the prohormone brain natriuretic peptide; CK — creatinine kinase; CK-MB - CK MB isoenzyme; EF - ejection fraction, LVDd - left ventricle end diastolic dimension; RVDd — right ventricle end diastolic dimension; LAD — left atrium diameter; IVSd — intraventricular septum end-diastolic diameter; PWDd — posterior wall end-diastolic diameter; TAPSE — tricuspid annular plane systolic excursion; IVC — inferior vena cava; VHD — valvular heart disease; AR — aortic regurgitation; AS — aortic stenosis; MR — mitral regurgitation; MS — mitral stenosis; TR — tricuspid regurgitation; TS — tricuspid stenosis; PR — pulmonary regurgitation; PS — pulmonary stenosis

The multivariable model for nocturnal fall in SBP based on laboratory, TTE and ECG parameters was:

$$
\begin{gathered}
\text { Nocturnal fall of SBP }[\%]=-27.28+1.47 \times H G B \\
-0.14 \times C K-M B+0.14 \times H R_{\max }
\end{gathered}
$$

According to the presented model, if a patient's hemoglobin concentration (HGB), creatinine kinase MB isoenzyme $(\mathrm{CK}-\mathrm{MB})$ or maximal heart rate $\left(\mathrm{HR}_{\max }\right)$ increases by 1 unit, nocturnal fall in SBP increases by $1.47 \%$ [95\% CI: 0.447 to 2.487 ), decreases by $0.14 \%$ (95\% CI: -0.271 to -0.002 ) or increases by $0.14 \%$ (95\% CI: 0.051 to 0.223 ), respectively (Tab. V).

The multivariable model for CBPP based on clinical factors is presented in Table VI. According to that model, if patient uses: $\beta$ - or $\alpha$-adrenolytics or 
Table III. 24-hour ECG Holter parameters

\begin{tabular}{|c|c|c|c|c|}
\hline & Dipper (29 pts) & Non-dipper (52 pts) & Reverse-dipper (22 pts) & \\
\hline Number of beats & $77498.38 \pm 16268.83$ & $75538.67 \pm 18449.94$ & $82990.00 \pm 24477.60$ & $p=0.89$ \\
\hline $\mathrm{HR}_{\text {mean }}$ & $66.66 \pm 7.33$ & $68.08 \pm 15.63$ & $65.14 \pm 6.34$ & $p=0.8$ \\
\hline $\mathrm{HR}_{\max }$ & $109.45 \pm 17.21$ & $102.50 \pm 18.43$ & $95.77 \pm 12.91$ & $p=0.02$ \\
\hline $\mathrm{HR}_{\text {min }}$ & $51.31 \pm 6.87$ & $52.56 \pm 6.78$ & $52.86 \pm 6.06$ & $p=0.65$ \\
\hline AF & $7.08 \pm 38.11$ & $7.84 \pm 27.14$ & $9.09 \pm 29.42$ & $p=0.71$ \\
\hline Pause & $0.24 \pm 0.83$ & $2.35 \pm 11.32$ & $1.36 \pm 4.01$ & $p=0.78$ \\
\hline Pause max (s) & $0.24 \pm 0.74$ & $0.43 \pm 1.11$ & $0.30 \pm 0.78$ & $p=0.78$ \\
\hline Bradycardias & $0.14 \pm 0.74$ & $1.38 \pm 4.60$ & $0.14 \pm 0.64$ & $p=0.13$ \\
\hline VT & $0.03 \pm 0.19$ & $0.31 \pm 1.55$ & $0.05 \pm 0.21$ & $p=0.5$ \\
\hline ExV & $482.48 \pm 2274.48$ & $426.31 \pm 1263.96$ & $259.68 \pm 722.38$ & $p=0.07$ \\
\hline $\mathrm{ExV} / \mathrm{h}$ & $70.03 \pm 258.05$ & $54.58 \pm 132.47$ & $41.18 \pm 101.26$ & $p=0.05$ \\
\hline $\mathrm{ExV} / \mathrm{min}$ & $3.31 \pm 8.70$ & $5.19 \pm 8.47$ & $4.91 \pm 5.13$ & $p=0.03$ \\
\hline Couples & $1.66 \pm 8.91$ & $0.92 \pm 3.09$ & $14.82 \pm 67.05$ & $p=0.02$ \\
\hline Triplets & $0.03 \pm 0.19$ & $0.06 \pm 0.31$ & $0.05 \pm 0.21$ & $p=0.98$ \\
\hline Bigeminy & $16.76 \pm 79.00$ & $7.31 \pm 26.85$ & $0.82 \pm 2.44$ & $p=0.78$ \\
\hline ExSV & $120.97 \pm 426.45$ & $180.27 \pm 408.43$ & $1158.27 \pm 2947.52$ & $p=0.05$ \\
\hline SVT & $1.52 \pm 4.36$ & $3.25 \pm 14.29$ & $39.64 \pm 176.11$ & $p=0.03$ \\
\hline ExSV/h & $27.17 \pm 104.68$ & $27.40 \pm 63.17$ & $142.86 \pm 321.07$ & $p=0.07$ \\
\hline ExSV/min & $4.59 \pm 7.11$ & $5.40 \pm 8.14$ & $10.50 \pm 13.91$ & $p=0.05$ \\
\hline Couples & $1.64 \pm 3.84$ & $5.90 \pm 20.13$ & $82.77 \pm 335.52$ & $p=0.02$ \\
\hline $\mathrm{P}[\mathrm{ms}]$ & $84.86 \pm 22.58$ & $80.55 \pm 32.14$ & $65.91 \pm 39.36$ & $p=0.29$ \\
\hline $\mathrm{PO}[\mathrm{ms}]$ & $145.17 \pm 43.88$ & $149.71 \pm 56.68$ & $142.73 \pm 80.84$ & $p=0.75$ \\
\hline QRS [ms] & $98.28 \pm 26.02$ & $120.10 \pm 101.88$ & $102.86 \pm 23.45$ & $p=0.5$ \\
\hline ST [ms] & $138.21 \pm 137.70$ & $154.90 \pm 143.47$ & $154.55 \pm 51.34$ & $p=0.04$ \\
\hline ECG HR & $73.62 \pm 13.04$ & $69.60 \pm 15.28$ & $64.73 \pm 16.44$ & $p=0.05$ \\
\hline QTc [ms] & $445.89 \pm 73.36$ & $428.76 \pm 60.99$ & $436.19 \pm 39.26$ & $p=0.66$ \\
\hline
\end{tabular}

pts — patients; $p$ - overall p-value for 3-group comparison of means (ANOVA test) or distributions (Kruskal-Wallis test) for continuous variables; HR — heart rate; AF — atrial fibrillation; VT — ventricular tachycardia; exV — ventricular extrasystoles; exV/h — exV per hour; exV/min — exVper minute; exSV — supraventricular extrasystoles; SVT — supraventricular tachycardia; exSV/h — exSV per hour; exSV/min — exSV per minute; QTC - corrected QT

Table IV. Multivariable model for nocturnal fall in systolic blood pressure (SBP) based on clinical factors

\begin{tabular}{|l|c|c|}
\hline & $\begin{array}{c}\text { Linear regression coefficient } \\
(95 \% \text { Cl) }\end{array}$ & \\
\hline Age & $-0.108(-0.187$ to -0.029$)$ & $\mathrm{p}=0.008$ \\
\hline DCM & $-8.329(-15.836$ to -0.823$)$ & $\mathrm{p}=0.03$ \\
\hline PAD & $-5.951(-10.540$ to -1.361$)$ & $\mathrm{p}=0.01$ \\
\hline$\alpha$-adrenolytics & $-6.017(-10.010$ to -2.024$)$ & $\mathrm{p}=0.004$ \\
\hline
\end{tabular}

torasemide, odds ratios of being in non-dipper or reverse dipper group versus dipper group or being in reverse dipper group versus dipper and non-dipper
Table V. Multivariable model for nocturnal fall in systolic blood pressure (SBP) based on laboratory, transthoracic echocardiography (TTE) and electrocardiography (ECG) parameters

\begin{tabular}{|l|c|c|}
\hline & $\begin{array}{c}\text { Linear regression coefficient } \\
\text { (95\% Cl) }\end{array}$ & \\
\hline HGB & $1.467(0.447$ to 2.487$)$ & $p=0.006$ \\
\hline CK-MB & $-0.136(-0.271$ to -0.002$)$ & $p=0.05$ \\
\hline $\mathrm{HR}_{\max }$ & $0.137(0.051$ to 0.223$)$ & $\mathrm{p}=0.002$ \\
\hline
\end{tabular}

Variables included in multivariable model for nocturnal fall in SBP based on all other assessed factors. $\mathrm{Cl}$ - confidence interval; $\mathrm{HGB}$ - hemoglobin; CK-MB — CK MB isoenzyme; HR - heart rate

group were 3.26 (95\% CI: 1.337 to 8.280 ), 5.80 (95\% CI: 1.678 to 22.110$)$ and 8.69 (95\% CI: 1.976 to 48.034$)$, respectively. 
Table VI. Multivariable model for circadian blood pressure pattern (CBPP) based on clinical factors

\begin{tabular}{|l|c|c|}
\hline & Proportional OR (95\% CI) & \\
\hline$\beta$-adrenolytics & $3.264(1.337$ to 8.280$)$ & $\mathrm{p}=0.01$ \\
\hline$\alpha$-adrenolytics & $5.799(1.678$ to 22.110$)$ & $\mathrm{p}=0.007$ \\
\hline Torasemide & $8.686(1.976$ to 48.034$)$ & $\mathrm{p}=0.007$ \\
\hline
\end{tabular}

Variables included in multivariable model for CBPP based on clinical factors. OR — odds ratio; $\mathrm{Cl}$ - confidence interval

\section{Discussion}

Millar-Craig et al. described circadian variation of BP in 1978 [9]. They used continuous intra-arterial monitoring. Nowadays ABPM is a noninvasive method to obtain CBPP [10]. Others proved that non-dipping pattern in hypertensive individuals might be associated with increased cardiovascular risk [6]. The lower or lack of fall in nocturnal BP could also cause target organ damage. Literature brings evidence that non-dipping blood pressure profile relates to left ventricle hypertrophy, cardiac functional alternations, renal damage, carotid artery abnormalities and cerebrovascular diseases [11-14].

Our study demonstrated that CBPP could be predicted according to clinical factors as well as laboratory, TTE and ECG parameters. The multivariable model for nocturnal fall in SBP based on clinical factors included age, diagnosis of DCM or PAD and the use of $\alpha$-adrenolytics. Others described lower sleep-time decline in SBP in older patients and it is known that older patients are more likely to be non-dippers or reverse dippers $[15,16]$. Some authors report that dipping status in hypertension influences cardiac structure and function $[13,17,18]$. Although, other studies failed to show statistically significant differences in cardiac structural or functional alternations between dippers and non-dippers [19]. We did not find any associations between CBPP and DCM. Wyss et al. showed hypertension as cardiovascular risk factor, though not associated with severity of PAD in multivariate models [20]. However, Vasunta et al. proved that non-dipping status in hypertension was a risk factor for early atherosclerosis development (intima-media thickness assessment) [21]. In other studies, association between non-dipping pattern of hypertension and early stages of atherosclerosis was also proven and influence of study population age was also emphasized [22]. The fact that the patients who took $\alpha$-adrenolytics were more likely to have lower fall in nocturnal BP could be explained by the fact that this class of drugs reduces nightly $\mathrm{BP}$ and restores dipping CBPP in non-dippers [23]. On the other hand, Pickering et al. showed that doxazosin lowered mostly morning BP and had relatively little influence on nightly BP [24]. According to the current guidelines of arterial hypertension management, $\beta$-blockers are recommended as antihypertensive drugs [25]. However, no studies comparing bedtime and morning administration of $\beta$-blockers have been conducted so far. These drugs are well known to influence daily BP, with minor influence on nightly BP [26]. There are only a few studies assessing influence of diuretics on dipping pattern. Uzu and Kimura showed that despite the fact that hydrochlorothiazide did not change CBPP in dippers, it changed non-dipping into dipping pattern in salt-sensitive hypertensive patients [27]. We did not find any associations between torasemide administration and CBPP. Furthermore, there is still little data comparing furosemide versus torasemide or other loop diuretics in the treatment of hypertension as well as there is little evidence upon which to base dosing of loop diuretics in the treatment of hypertension. We found no data analyzing different loop diuretics or different groups of diuretics in the context of hypertension dipping status [28].

Among laboratory, TTE and ECG parameters, $\mathrm{HGB}, \mathrm{CK}-\mathrm{MB}$ and $\mathrm{HR}_{\max }$ seemed to have influence on nocturnal fall in SBP. We could not find any associations between HGB parameters and CBPP. Though lower HGB might be explained with both higher prevalence and severity of CKD among patients with lower decline in nocturnal SBP and higher prevalence of non-dipping pattern of hypertension among patients with CKD [29, 30]. In our study, there was higher prevalence of CKD among non-dippers and reversedippers compared with dippers. With the worsening kidney function we observed lower HGB concentration [31]. Non-dipping patients are described to have impairment in autonomic nervous drive (especially parasympathetic inactivity) [32]. No studies regarding either $\mathrm{HR}_{\text {max }}$ or CK-MB and CBPP were found.

As both, the strength and the limitation of the study we see a relatively big subgroup of reversedipper patients in our study population. It may be considered strength, since data regarding reverse-dippers are mostly lacking, and this group of patients is frequently omitted in other studies. Though, it may be seen also as limitation, because we had no data to compare our outcomes. Further limitation of our study could be the fact that we divided patients based only on one ABPM outcome. We also included relatively small group of patients in the study.

\section{Conclusions}

We found that detailed medical history could help us to predict not only nocturnal fall in SBP, but also patient's CBPP. These findings may have influence 
on care of patients with hypertension, though further studies are needed.

\section{Disclosure of interest \\ The authors report no conflicts of interest.}

\section{References}

1. Kearney PM, Whelton M, Reynolds K, et al. Global burden of hypertension: analysis of worldwide data. Lancet. 2005; 365(9455): 217-223, doi: 10.1016/S0140-6736(05)17741-1, indexed in Pubmed: 15652604

2. Guilbert JJ. The world health report 2002 — reducing risks, promoting healthy life. Educ Health (Abingdon). 2003; 16(2): 230, doi: 10 $.1080 / 1357628031000116808$, indexed in Pubmed: 14741909.

3. Timio M, Venanzi S, Lolli S, et al. "Non-dipper" hypertensive patients and progressive renal insufficiency: a 3-year longitudinal study. Clin Nephrol. 1995; 43(6): 382-387, indexed in Pubmed: 7554522.

4. Suzuki M, Guilleminault C, Otsuka K, et al. Blood pressure "dipping" and "non-dipping" in obstructive sleep apnea syndrome patients. Sleep. 1996; 19(5): 382-387, doi: 10.1093/sleep/19.5.382, indexed in Pubmed: 8843529.

5. Hermida RC, Crespo JJ, Otero A, et al. Asleep blood pressure: significant prognostic marker of vascular risk and therapeutic target for prevention. Eur Heart J. 2018; 39(47): 4159-4171, doi: 10.1093/ eurheartj/ehy475, indexed in Pubmed: 30107515.

6. Verdecchia P, Schillaci G, Reboldi G, et al. Ambulatory blood pressure. An independent predictor of prognosis in essential hypertension. Hypertension. 1994; 24(6): 793-801, doi: 10.1161/01. hyp.24.6.793, indexed in Pubmed: 7995639.

7. Staessen JA, Bieniaszewski L, O’Brien E, et al. Nocturnal blood pressure fall on ambulatory monitoring in a large international database. The „Ad Hoc” Working Group. Hypertension. 1997; 29 (1 Pt 1):3039, doi: 10.1161/01.hyp.29.1.30, indexed in Pubmed: 9039076.

8. Team RCR. Team RCR. A language and environment for statistical computing. R Foundation for Statistical Computing. Vienna 2012: ISBN 3-900051-07-0; 2014.

9. Millar-Craig MW, Bishop CN, Raftery EB. Circadian variation of blood-pressure. Lancet. 1978; 1(8068): 795-797, doi: 10.1016/ s0140-6736(78)92998-7, indexed in Pubmed: 85815.

10. Pickering TG, Hall JE, Appel LJ, et al. Council on High Blood Pressure Research Professional and Public Education Subcommittee, American Heart Association. Recommendations for blood pressure measurement in humans: an AHA scientific statement from the Council on High Blood Pressure Research Professional and Public Education Subcommittee. J Clin Hypertens (Greenwich). 2005; 7(2): 102-109, indexed in Pubmed: 15722655.

11. Cuspidi C, Meani S, Salerno M, et al. Cardiovascular target organ damage in essential hypertensives with or without reproducible nocturnal fall in blood pressure. J Hypertens. 2004; 22(2): 273-280, doi: 10.1097/00004872-200402000-00010, indexed in Pubmed: 15076184.

12. Cuspidi C, Michev I, Meani S, et al. Reduced nocturnal fall in blood pressure, assessed by two ambulatory blood pressure monitorings and cardiac alterations in early phases of untreated essential hypertension. J Hum Hypertens. 2003; 17(4): 245-251, doi: 10.1038/ sj.jhh.1001546, indexed in Pubmed: 12692569.

13. Ijiri H, Kohno I, Yin D, et al. Cardiac arrhythmias and left ventricular hypertrophy in dipper and nondipper patients with essential hypertension. Jpn Circ J. 2000; 64(7): 499-504, doi: 10.1253/jcj.64.499, indexed in Pubmed: 10929777.

14. Passino C, Magagna A, Conforti F, et al. Ventricular repolarization is prolonged in nondipper hypertensive patients: role of left ventricular hypertrophy and autonomic dysfunction. J Hypertens. 2003; 21(2): 445-451, doi: 10.1097/00004872-200302000-00038, indexed in Pubmed: 12569277.

15. Hermida RC, Ayala DE, Crespo JJ, et al. Influence of age and hypertension treatment-time on ambulatory blood pressure in hypertensive patients. Chronobiol Int. 2013; 30(1-2): 176-191, doi: 10.3109/0 7420528.2012.701131, indexed in Pubmed: 23077974.

16. Di Iorio A, Marini E, Lupinetti M, et al. Blood pressure rhythm and prevalence of vascular events in hypertensive subjects. Age Ageing. 1999; 28(1): 23-28, doi: 10.1093/ageing/28.1.23, indexed in Pubmed: 10203200

17. Verdecchia P, Schillaci G, Borgioni C, et al. Circadian blood pressure changes and left ventricular hypertrophy in essential hypertension. Circulation. 1990; 81(2): 528-536, doi: 10.1161/01.cir.81.2.528, indexed in Pubmed: 2137047.

18. Balci B, Yilmaz O, Yesildag O. The influence of ambulatory blood pressure profile on left ventricular geometry. Echocardiography. 2004; 21(1): 7-10, indexed in Pubmed: 14717714.

19. Cuspidi C, Michev I, Meani S, et al. Non-dipper treated hypertensive patients do not have increased cardiac structural alterations. Cardiovasc Ultrasound. 2003; 1: 1, indexed in Pubmed: 12709263.

20. Wyss TR, Adam L, Haynes AG, et al. Impact of cardiovascular risk factors on severity of peripheral artery disease. Atherosclerosis. 2015; 242(1): 97-101, doi: 10.1016/j.atherosclerosis.2015.07.002, indexed in Pubmed: 26186656.

21. Vasunta RL, Kesäniemi YA, Ylitalo A, et al. Nondipping pattern and carotid atherosclerosis in a middle-aged population: OPERA Study. Am J Hypertens. 2012; 25(1): 60-66, doi: 10.1038/ajh.2011.159, indexed in Pubmed: 21866184.

22. Cuspidi C, Macca G, Sampieri L, et al. Target organ damage and nondipping pattern defined by two sessions of ambulatory blood pressure monitoring in recently diagnosed essential hypertensive patients. J Hypertens. 2001; 19(9): 1539-1545, doi: 10.1097/00004872200109000-00004, indexed in Pubmed: 11564972.

23. Kario K, Schwartz JE, Pickering TG. Changes of nocturnal blood pressure dipping status in hypertensives by nighttime dosing of alphaadrenergic blocker, doxazosin : results from the HALT study. Hypertension. 2000; 35(3): 787-794, doi: 10.1161/01.hyp.35.3.787, indexed in Pubmed: 10720596.

24. Pickering TG, Levenstein M, Walmsley P. Nighttime dosing of doxazosin has peak effect on morning ambulatory blood pressure. Results of the HALT Study. Hypertension and Lipid Trial Study Group. Am J Hypertens. 1994; $7(9$ Pt 1): 844-847, doi: 10.1093/ ajh/7.9.844, indexed in Pubmed: 7811444

25. Mancia G, Fagard R, Narkiewicz K, et al. 2013 ESH/ESC guidelines for the management of arterial hypertension: the Task Force for the Management of Arterial Hypertension of the European Society of Hypertension (ESH) and of the European Society of Cardiology (ESC). Eur Heart J. 2013; 34(28): 2159-2219, doi: 10.1093/ eurheartj/eht151, indexed in Pubmed: 23771844.

26. Stanton A, O'Brien E. Auswirkungen der Therapie auf das zirkadiane Blutdruckprofil. Kardio. 1994; 3: 1-8.

27. Uzu T, Kimura G. Diuretics shift circadian rhythm of blood pressure from nondipper to dipper in essential hypertension. Circulation. 1999; 100(15): 1635-1638, doi: 10.1161/01.cir.100.15.1635, indexed in Pubmed: 10517735.

28. Malha L, Mann SJ. Loop Diuretics in the Treatment of Hypertension. Curr Hypertens Rep. 2016; 18(4): 27, doi: 10.1007/s11906-0160636-7, indexed in Pubmed: 26951244.

29. Hermida RC, Ayala DE, Smolensky MH, et al. Chronotherapy improves blood pressure control and reduces vascular risk in CKD. Nat Rev Nephrol. 2013; 9(6):358-368, doi: 10.1038/nrneph.2013.79, indexed in Pubmed: 23609565.

30. Agarwal R, Kariyanna SS, Light RP. Circadian blood pressure classification scheme and the health of patients with chronic kidney disease. Am J Nephrol. 2009; 30(6): 536-546, doi: 10.1159/000252774, indexed in Pubmed: 19844085.

31. Panjeta M, Tahirovic I, Karamehic J, et al. The Relation of Erythropoietin Towards Hemoglobin and Hematocrit in Varying Degrees of Renal Insufficiency. Mater Sociomed. 2015; 27(3): 144-148, doi: $10.5455 / \mathrm{msm} .2015 .27 .144-148$, indexed in Pubmed: 26236158.

32. Hojo Y, Noma S, Ohki T, et al. Autonomic nervous system activity in essential hypertension: a comparison between dippers and nondippers. J Hum Hypertens. 1997; 11(10): 665-671, indexed in Pubmed: 9400909. 